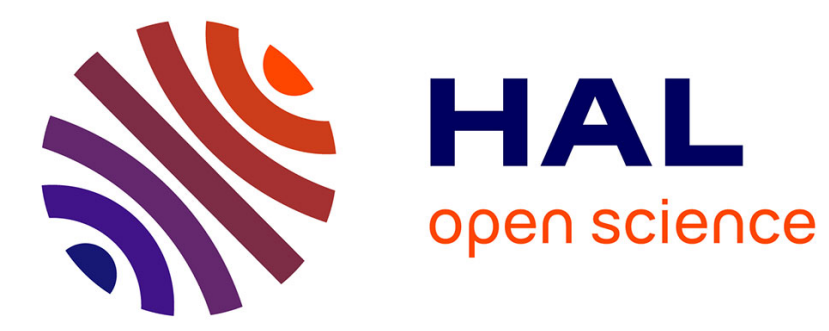

\title{
Organisation frontière et maintien institutionnel. Le cas du Comité permanent amiante en France
}

Hélène Peton

\section{To cite this version:}

Hélène Peton. Organisation frontière et maintien institutionnel. Le cas du Comité permanent amiante en France. Revue Française de Gestion, 2011, pp.176. 10.3166/rfg.217.117-135 . hal-01130486

\author{
HAL Id: hal-01130486 \\ https://hal.science/hal-01130486
}

Submitted on 11 Mar 2015

HAL is a multi-disciplinary open access archive for the deposit and dissemination of scientific research documents, whether they are published or not. The documents may come from teaching and research institutions in France or abroad, or from public or private research centers.
L'archive ouverte pluridisciplinaire HAL, est destinée au dépôt et à la diffusion de documents scientifiques de niveau recherche, publiés ou non, émanant des établissements d'enseignement et de recherche français ou étrangers, des laboratoires publics ou privés. 
Organisation Frontière et Maintien institutionnel

Le cas du Comité Permanent Amiante en France

\author{
Hélène Peton \\ Université Paris-Dauphine \\ Dauphine Recherches en Management (DRM) \\ Place du Maréchal de Lattre de Tassigny \\ 75775 Paris Cedex 16 \\ helene.peton@dauphine.fr
}

\title{
Résumé
}

Comment mettre en échec un mouvement social ? Nous étudions le rôle des organisations frontière dans le travail de maintien institutionnel. Notretravail s'appuie sur le cas de l'amiante en France à travers la mise en place du CPA (Comité Permanent Amiante). Le CPA (1982-1995) est une organisation frontière qui a promu l'Usage Contrôlé de l'Amiante et a ainsi participé au maintien de la pratique institutionnalisée.

\section{Summary}

How to defeat a social movement? We focus on the role of boundaries organizations in the institutional work engaged in the logics of maintenance. We study the case of the CPA (Asbestos Permanent Committee) as a boundary organization in the process of maintenance of an institutionalized practice through the 'controlled use of asbestos'. 


\section{INTRODUCTION}

Depuis quelques années, la question du changement a connu un intérêt grandissant dans la littérature néo-institutionnelle. Cette littérature sur le changement a permis de mettre en évidence la capacité des acteurs à agir au sein de l'institution. Les acteurs, pour défendre leurs intérêts produisent intentionnellement des efforts. Le concept de travail institutionnel (Lawrence etSuddaby, 2006) correspond aux actions menées par les acteurs (individuels ou collectifs) pour créer, détruire mais aussi maintenir une institution. Les institutions peuvent être définies comme des règles et des significations partagées qui permettent de définir les relations sociales et aident à légitimer qui occupe quelle position dans ces relations (Fligstein, 2001). Ainsi, les pratiques institutionnalisées sont des vecteurs de l'institution. Les pratiques façonnent les comportements en définissant ce qu'il faut faire ou non de telle sorte que s'en éloigner est coûteux pour les acteurs. Elles sont inscrites dans des routines et font partie de la construction sociale du champ (Jepperson, 1991). Comme le souligne Zucker (1987), ces pratiques sont maintenues sur un temps long sans qu'il n'y ait besoin de le justifier et sont très résistantes au changementElles peuvent donc être l'objet d'un travail institutionnel qui ferait évoluer ces pratiques ou les maintiendrait. Dès lors des luttes institutionnelles apparaissent pour maintenir la pratique (defensivework) ou la détruire (disruptive work) (Maguire et Hardy, 2009). La contestation menée par un mouvement social s'inscrit tout à fait dans cette perspective de luttes institutionnelles. Cependant, les moments de trêves issus d'une collaboration (O’Mahony et Bechky, 2008) sont également des lieux de travail institutionnel autour de la défense d'intérêts convergents ou divergents. Nous introduisons dans cet article le concept d'organisation frontière pour comprendre comment la collaboration permise par cette structure est une réponse envisageable à une sortie de crise qui mettrait en échec un mouvement social. Issu de la littérature en sociologie des sciences, le concept d'organisation frontière s'intéresse à des organisations intermédiaires mises en place pour réunir des acteurs 
de différentes communautés (en particuliers des acteurs scientifiques et politiques). Tout l'enjeu du bon fonctionnement de cette organisation repose sur la capacité des acteurs à trouver des intérêts convergents afin de défendre un objectif commun (Guston, 2001). Cette forme organisationnelle repose sur la volonté de rassembler des acteurs tout en leur permettant de conserver leur identité et leurs propres intérêts divergents (O’Mahony et Bechky, 2008).

Nous nous focalisons sur l'impact d'une organisation frontière comme réponse à une situation de luttes institutionnelles. Notre travail s'articulera alors autour de deux questions : comment l'organisation frontière peut-elle être une réponse aux stratégies disruptives menées par les mouvements sociaux ? En quoi cette organisation participe-t-elle au maintien de la pratique institutionnalisée ? Afin de répondre aux questions théoriques soulevées précédemment, nous étudierons le cas de l'amiante en France. La France a connu une histoire particulière avec l'amiante, ce qui explique aussi l'ampleur du scandale qui a éclaté dans les années 1990. Son interdiction officielle, le $1^{\mathrm{er}}$ janvier 1997, arrive avec quelques années de retard par rapport à d'autres pays européens. Ce retard est dû à une période de maintien institutionnel entre 1982 et 1995. Suite à une mobilisation à la fin des années 70, la France a décidé de mettre en place une organisation frontière, le CPA (Comité Permanent Amiante) qui a mis en place et diffusé une nouvelle doctrine : l'usage contrôlé de l'amiante. Dans la première partie théorique, nous définirons les concepts de travail institutionnel et nous montrerons en quoi le concept d'organisation frontière peut être une solution pour mettre en échec un mouvement social. Puis, nous montrerons dans quelle mesure l'organisation frontière peut être une organisation au service du maintien institutionnel. Dans un second temps, nous présenterons notre méthodologie qui repose sur une étude de cas unique d'un processus à partir de données secondaires variées et d'entretiens avec les acteurs clef du champ. Nous présenterons rapidement les grandes phases de l'amiante en France pour ensuite nous concentrer plus 
particulièrement sur la période marquée par la présence du CPA. La dernière partie formule les principales conclusions de notre recherche en ouvrant également quelques perspectives de recherche.

\section{CADRE THEORIQUE}

Les institutions peuvent être définies comme des règles et des significations partagées qui permettent de définir les relations sociales et aident à légitimer qui occupe quelle position dans ces relations (Fligstein, 2001). Il s'agit comme le soulignent Barley et Tolbert (1997, p.99) d'une accumulation de pratiques et de codes partagés qui permettent d'avoir les clefs pour imposer une pratique comme légitime. Chaque pratique institutionnalisée donne ainsi le cadre pour les interactions futures. L'étude des institutions se fait au niveau du champ qui est constitué de l'ensemble des acteurs qui sont concernés par une problématique commune et qui de ce fait interagissent particulièrement les uns avec les autres. Les logiques institutionnelles jouent alors un rôle central dans la définition du champ et de son fonctionnement. Pour le dire simplement, nous pouvons reprendre les propos de Jackall (1988: 112): «An institutionallogicis the way a particular social world works ». Cela se traduit par des règles, des récompenses et des sanctions que les acteurs élaborent et transforment pour être en conformité avec le champ. La conformité avec le champ se traduit par les pratiques auxquelles se livrent les acteurs. Les pratiques institutionnalisées sont des comportements partagés qui relèvent du domaine de la routine (Whittington, 2006). Ces pratiques participent à la diffusion des éléments cognitifs, normatifs et régulatifs quiassurent la stabilité et le sens de la vie sociale (Scott, 2001: 48). Comme le souligne Barnes (2001), ces pratiques sont définies par le groupe et lui appartiennent. Les membres de ce groupe les intègrent et les reproduisent. Elles sont donc prises pour acquises et il est souvent difficile pour les acteurs de les questionner. Le travail de Maguire et Hardy (2009)sur l'usage du DDT met justement en évidence le travail discursif engagé par les acteurs pour questionner ces pratiques. Le DDT est un pesticide 
reconnu et efficace. Son usage s'est très vite diffusé (parfois au-delà même du domaine de l'agriculture), devenant une norme dans le traitement des cultures. Il aura fallu un choc externe par la publication du pamphlet «SilentSpring » pour que son usage soit contesté. Il est donc nécessaire de faire des efforts pour faire évoluer une pratique institutionnalisée. Le travail de Maguire et Hardy (2009) a également permis de mettre en évidence l'existence d'un véritable travail de maintien de la pratique institutionnalisée. Cette notion de travail de maintien s'inscrit dans une nouvelle perspective du maintien institutionnel qui n'est pas uniquement expliqué par des mécanismes inertiels (Barley et Tolbert, 1997 ; Scott, 2001). Lawrence et Suddaby (2006) proposent le concept de travail institutionnel pour rendre compte de ces efforts. La notion de travail institutionnel fait référence aux actions volontaires des individus et des organisations pour créer, maintenir ou détruire une institution (Lawrence et al., 2009). Ce travail institutionnel peut se traduire par des efforts pour modifier la reconnaissance et l'usage de ces routines. Zietsma et Lawrence (2010) définissent ainsi le travail institutionnel engagé dans le changement de pratique ( practice work»). Les pratiques institutionnalisées participent à la définition et à la perception du champ institutionnel. Les frontières de l'institution permettent de définir un ensemble de pratiques légitimes et les acteurs peuvent s'engager dans un travail visant à établir, renforcer ou détruire les frontières institutionnelles (Arndt et Bigelow, 2005). Les organisations frontières s'inscriventdans ce travail sur les frontières institutionnelles (Lawrence et Hardy, 1999 ;O’Mahony et Bechky, 2008). Les organisations frontières ont été étudiées en sociologie des sciences comme des organisations intermédiaires créées pour faciliter la collaboration entre scientifiques et non scientifiques. Ces organisations réunissent des acteurs appartenant à des communautés différentes qui ne pourraient pas aussi bien collaborer sans cette structure qui par son indépendance leur permet de maintenir leur identité tout en défendant un objectif commun (Guston, 2001). Il est donc possible de faire collaborer au sein de cette structure des 
acteurs aux intérêts divergents puisque la construction de cette structure repose sur cette volonté de rassembler ces acteurs (O’Mahony et Bechky, 2008). La difficulté de ces organisations est de réussir à redéfinir les frontières du champ en permettant des passerelles entre ces acteurs. Ces organisations frontières sont donc des lieux de collaboration et offrent un nouveau regard sur la dynamique des mouvements sociaux. En étudiant la communauté de l'open-source face aux entreprises du marché, les auteurs soulignent la possibilité pour des acteurs précédemment en conflit de trouver des intérêts convergents tout en conservant leur propre identité et leurs propres intérêts. Chaque groupe bénéficie de cette organisation en ayant accès à des ressources complémentaires des siennes (en l'occurrence les membres de la communauté open-source voulaient élargir les possibilités de diffusion de leur système et bénéficier des ressources mises à disposition par les entreprises de l'organisation frontière ; en retour, les entreprises ont bénéficié de l'expertise technique des membres de la communauté open-source notamment par l'accès à des codes et se sont positionnées sur un marché qu'ils pensent être porteur d'avenir). La littérature sur les mouvements sociaux a plutôt eu tendance à se focaliser sur les questions relatives à l'émergence d'un mouvement et moins aux résultats de ces mouvements (Giugni, 1998).Les organisations frontières sont des moments de collaboration, de trêves dans le processus de luttes institutionnelles opposants les mouvements sociaux à l'institution. Ainsi, Snow, Soule et Kriesi (2004) définissent le mouvement social comme des challengers ou des défenseurs d'une autorité institutionnelle existante. Nous nous intéresserons plus particulièrement au moment du passage à la collaboration. Nous nous focalisons sur l'impact d'une organisation frontière comme réponse à une situation de luttes institutionnelles. Notre travail s'articulera alors sur deux questions : comment l'organisation frontière peut-elle être une réponse aux stratégies disruptives menées par les mouvements sociaux? En quoi cette organisation participe-t-elle au maintien de la pratique institutionnalisée? 


\section{METHODOLOGIE ET SOURCES DE DONNEES}

La méthodologie envisagée consiste en une étude de cas unique et longitudinale (Yin, 2003). Nous avons dans un premier temps retracé la chronologie des évènements liés à l'histoire de l'amiante en France à l'aide de données secondaires adaptées aux différentes étapes du processus. Nous avons collecté les données sur la base de différentes sources : (1) des rapports officiels (Cour de cassation, SENAT, Assemblée Nationale, Cour des comptes, etc.) ; (2) des documents officiels produits par les organismes de prévention (notamment I'INRS pour les compte rendus et publications du CPA) ; (3) les ouvrages et articles de recherche déjà publiés sur le sujet ; (4) de données d'archives audiovisuelles et issues de la presse quotidienne française; et (5) des archives du syndicat CGT, du collectif de Jussieu. Dans un second, temps nous avons réalisé des entretiens semi-directifs en face à face avec les acteurs du champ. Nous avons entre autre rencontré des représentants d'association, des victimes, des avocats, des journalistes et des anciens membres du CPA. Tous ces acteurs ont participé activement à la vie de l’amiante en France.

\section{Contexte empirique}

\section{L'amiante en France : une rapide présentation}

L'amiante est une roche fibreuse dotée de qualités d'isolation et de résistance. Son faible coût explique également son utilisation massive dans des secteurs très variés (construction, industrie automobile ou encore l'industrie textile) ${ }^{1}$. Le mythe de l'amiante prendra réellement forme lors de son industrialisation massive au début du $20^{\text {ème }}$ siècle. Comme le souligne François Malye (1995, p29) :«Dès lors, lorsqu'on pense chaleur ou incendie, la réaction est de répondre «amiante». En cette fin de siècle, les dés sont jetés : ce minéral sera le compagnon de route du capitalisme industriel » selon la belle formule du Collectif antiamiante de Jussieu en 1977 ».La France abandonne rapidement son activité de production ${ }^{2}$.

\footnotetext{
${ }^{1}$ Dans les années 70, l'amiante est présent dans plus de 3000 produits de consommation courante.

${ }^{2}$ Les mines de Canari en Corse ferment en 1965
} 
Deux grands groupes français à dimension internationale se sont spécialisés dans l'activité de transformation : Eternit et Saint-Gobain. De 1945 au milieu des années 1970, le tonnage d'amiante importé en France ne cesse d'augmenter. Le pic d'importation est de 156000 tonnes en 19753. En 1996, la France importait 35000 tonnes et uniquement 12 tonnes en 1999, soit juste 2 ans après l'interdiction. Ces chiffres font de la France un des pays ayant le plus utilisé l'amiante (dans les années 1970, la France est le deuxième importateur mondial d'amiante).

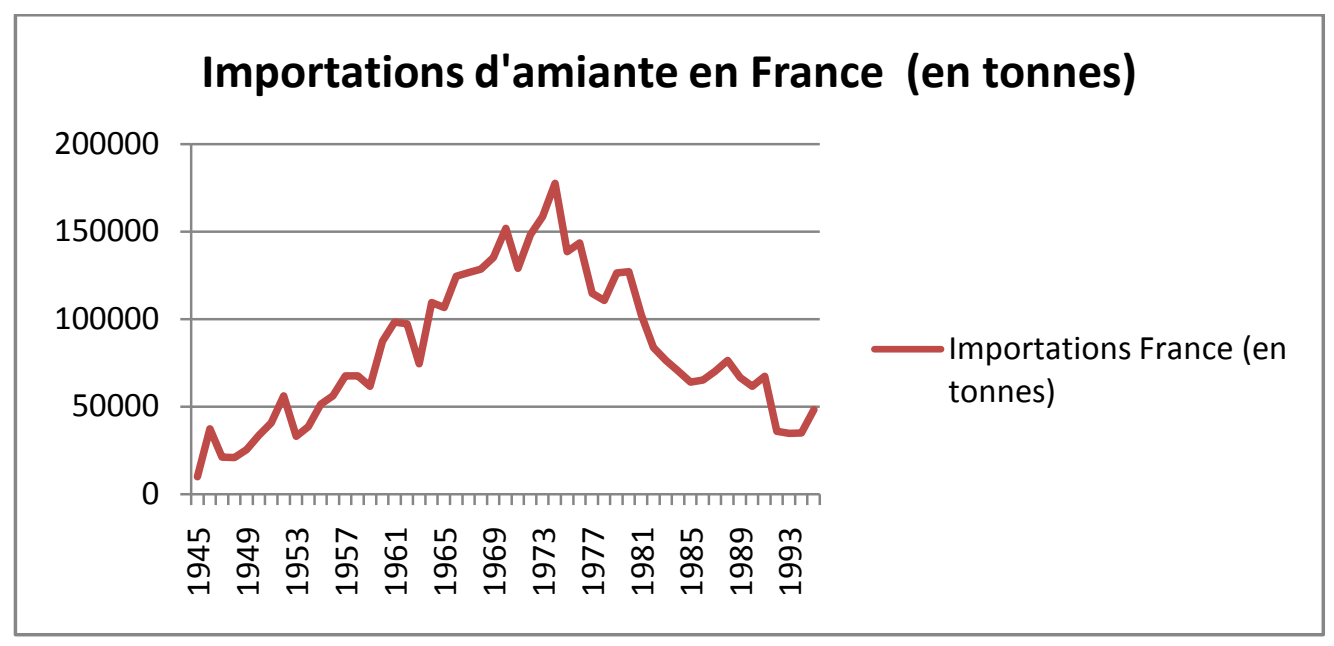

Figure 1 : Importations françaises d’amiante en tonnes par années (Source : Ministère du Commerce Extérieur, France), données reprises sur le site de l'ANDEVA

La France a connu une histoire particulière avec l'amiante, ce qui explique aussi l'ampleur du scandale dans les années 1990. Son interdiction officielle, le $1^{\text {er }}$ janvier 1997, arrive avec quelques années de retard par rapport à d'autres pays européens ${ }^{3}$ : le rapport publié par le Sénat (Dériot et Godefroy, 2005, p. 41), reprenant les propos du Professeur Gott, souligne à ce titre que la France a «mal géré le risque lié à l'amiante» en comparaison d'autres pays comme l'Angleterre, l'Allemagne ou les Etats-Unis. La France est surtout à l'origine de l'Usage Contrôlé de l'Amiante, doctrine développée dans les années 80 sous l'impulsion du

\footnotetext{
${ }^{3}$ A titre d'exemple : L'Allemagne, signe un accord volontaire avec l'industrie dès 1979 avec pour objectif de parvenir au bout de 10 ans à une interdiction de l'amiante. Le Danemark, interdit de produire, d'importer, d'utiliser et de travailler l'amiante ou tout produit contenant de l'amiante à compter du 1/1/1986. La Finlande interdit l'importation d'amiante et de produits contenant de l'amiante à compter du 1/1/1993.
} 
CPA (Comité Permanent Amiante), organisation frontière mise en place par les pouvoirs publics pour répondre à la première crise de l'amiante en France (fin des années 70). L'adoption de cette position a ralenti le processus d'interdiction de la pratique. Une comparaison de l'évolution des importations entre la France et les Etats-Unis (qui n'ont pas adopté cette position) permet d'illustrer ce point. En effet, la consommation d'amiante en France dans les années 80 (période du CPA) reste stable autour des 70000 tonnes consommées et chute brutalement au début des années 90 pour se stabiliser autour des 30000 tonnes. Aux Etats-Unis, la décroissance est beaucoup plus régulière et commence dès le début des années 80 (après les premières mesures de restriction).Ainsi, au début des années 80 , la consommation est de 380000 tonnes pour atteindre moins de 50000 tonnes en 1990. Cette décroissance suit une progression linéaire. Il y a donc bien eu un «effet CPA et Usage Contrôlé de l'Amiante» en France, effet qui a ralenti la désinstitutionalisation de la pratique $^{45}$.

\section{Les différentes phases de l'histoire de l'amiante en France}

Il nous semble dans un premier temps nécessaire de retracer rapidement les grandes étapes de 1'histoire de l'amiante en France (Figure 2).

\begin{tabular}{|c|c|c|c|c|}
\hline $1945-1970$ & 1970-1982 & 1982-1992 & 1992-1997 & $1997-\ldots$ \\
\hline $\begin{array}{l}\text { Age d'or de } \\
\text { l'amiante en } \\
\text { France }\end{array}$ & $\begin{array}{l}\text { Premiers } \\
\text { questionnements de } \\
\text { l'amiante }\end{array}$ & $\begin{array}{l}\text { Gestion de l'amiante } \\
\text { déléguée au Comité } \\
\text { Permanent Amiante }\end{array}$ & Rupture du consensus & $\begin{array}{l}\text { Interdiction } \\
\quad \text { au } 1^{\text {er }} \\
\text { janvier } 1997\end{array}$ \\
\hline & $\begin{array}{c}\text { EMERGENCE DU } \\
\text { PREMIER } \\
\text { MOUVEMENT }\end{array}$ & $\begin{array}{l}\text { USAGE CONTROLE } \\
\text { DE L'AMIANTE }\end{array}$ & $\begin{array}{c}\text { EMERGENCE DU } \\
\text { SECOND } \\
\text { MOUVEMENT }\end{array}$ & \\
\hline & $\begin{array}{l}\text { PREMIERE CRISE } \\
\text { DE L'AMIANTE }\end{array}$ & & $\begin{array}{r}\text { SECONDE CRISE DE } \\
\text { L'AMIANTE }\end{array}$ & \\
\hline
\end{tabular}

Figure 2Repères chronologiques et délimitation des différentes phases de l'histoire de l'amiante en France

\footnotetext{
${ }^{4}$ Des chiffres et graphiques sont disponibles sur le site de l'ANDEVA

${ }^{5}$ Il est aujourd'hui encore difficile de donner une estimation précise du nombre de morts et de malades liés à l'usage contrôlé de l'amiante. Pour l'instant les spécialistes s'accordent à dire que le retard pris dans la prévention pourrait conduire à un pic de pathologies dans les années 2020.
} 
La période 1945-1970 marque l'âge d'or de l'amiante en France et dans le monde. L'amiante devient incontournable. Ces qualités physiques d'isolation et son faible coût en font un minerai indispensable. La première période correspond aux années 1970. Premiers questionnements, premières victimes et premiers mouvements sociaux, le mythe de l'amiante est largement discuté. Tout commence par la grève des ouvrières de l'entreprise Amisol de Clermond-Ferrand. Amisol était une entreprise de transformation d'amiante. A l'annonce de la fermeture de l'usine en 1974, les ouvrières commencent une grève (qui durera 31 mois) pour obtenir la réouverture de leur usine. Durant cette grève, 12 ouvriers de l'usine meurent de maladies professionnelles liées à une exposition intense à l'amiante, et de nombreuses pathologies se déclarent. Parallèlement et de manière tout à fait indépendante, des professeurs de Jussieu constatent un dérèglement de leurs appareils de mesures. Ils remarquent alors la présence de poussières d'amiante dans leurs laboratoires et s'intéressent alors à cette fibre, présente dans tout le flocage de l'université. C'est en faisant leur enquête qu'ils entendent parler du cas d'Amisol. Une délégation, menée par le Professeur Pézerat, décide alors d'aller rencontrer ces ouvrières et surtout de leur faire part de leurs découvertes quant à la dangerosité de l'amiante. Le problème local et ouvrier prend alors une autre dimension: Jussieu est une université très connue et le collectif créé bénéficie d'une grande légitimité. Le savoir rencontre la preuve et réussit à susciter un intérêt médiatique. La mobilisation s'élargit alors : 1'UFC Que Choisir publie une enquête sur la présence d'amiante dans les filtres utilisés dans la production de vin et révèle la présence d'amiante dans plus de 3000 produits de consommation courante. Des associations de riverains et des associations de protection de la nature s'impliquent également dans la mobilisation (en particulier, la première association se crée à Condé- sur- Noireau suite à la pollution de la rivière jouxtant l'entreprise de transformation d'amiante Férodo). Ces luttes institutionnelles conduisent la prise de mesures par les pouvoirs publics, dont la mesure phare est l'interdiction du flocage en amiante dans 
tous les bâtiments. La seconde période commence avec la création du CPA (Comité Permanent Amiante) en 1982 et la mise en place de «l'usage contrôlé de l'amiante ». Après la confrontation, le temps est à la collaboration. Le dossier de l'amiante est confié pendant près de 10 ans à cette organisation frontière qui compte des représentants des industriels (producteurs ou utilisateurs d'amiante), des scientifiques (épidémiologistes et médecins du travail), des syndicats (sauf FO qui quittera le CPA en 1986) ${ }^{6}$. La dernière période marque la rupture de ce consensus. Cette rupture apparaît dans un contexte où les pressions internationales (en particulier européennes) se font de plus en plus fortes pour aller vers une interdiction de l'amiante. Le début des années 90 est également marqué par une succession d'évènements, au départ assez indépendants, mais qui ont créé la dynamique nécessaire au changement. Ainsi, les années 90 marquent l'apparition des pathologies, notamment chez les victimes environnementales ${ }^{7}$. Des veuves de professeurs d'un lycée de Gérardmer portent plainte contre X en 1992. Elles attribuent la mort de leur époux à la présence de flocage d'amiante sur leur lieu travail. Parallèlement, deux journalistes (François Malye et Patrick Herman) décident de mener des enquêtes sur le CPA et l'amiante. Ces enquêtes vont trouver un écho médiatique national et participer à la naissance du «scandale de l'air contaminé » ${ }^{8}$. C'est aussi à ce moment que le Professeur Pézerat, toujours à Jussieu, qui avait refusé de participer au CPA, trouve l'opportunité pour à nouveau faire entendre sa voix et remobiliser un collectif anti-amiante. Il fait venir à Jussieu Julian Peto, épidémiologiste britannique, qui publie des résultats et des prévisions très alarmants sur les morts et malades à venir pour les prochaines années en Grande-Bretagne (où des mesures beaucoup plus drastiques ont été prises). Sous l'impulsion du Professeur Pézerat, un groupe de scientifiques s'organise, s'appuie sur des avocats, et créent l'ANDEVA (Association Nationale des Victimes de

\footnotetext{
${ }^{6}$ Nous reviendrons plus en détail par la suite sur cette période. L'objectif ici est de présenter succinctement les différents périodes et leur enchaînement.

${ }^{7}$ Il faut environ une quarantaine d'années pour que les pathologies associées à l'amiante se déclarent.

${ }^{8}$ Pour reprendre le titre du livre de François Malye publié en 1996, "Amiante, le dossier de l'air contaminé »
} 
l'Amiante). Ils portent le dossier de l'amiante sur la scène politique et judiciaire pour obtenir son interdiction. Le compromis ne tient plus : les membres du CPA décident de ne plus y siéger ${ }^{9}$. Septembre 1995 marque la fin du CPA en France. En quelques mois, l'amiante est interdit en France ${ }^{10}$.

\begin{tabular}{|c|c|}
\hline 1975 & $\begin{array}{l}\text { Annonce de la fermeture de l'entreprise Amisol (entreprise transformatrice d'amiante de } \\
\text { Clermont-Ferrand). Création du "Collectif inter-syndical de sécurité des universités de Jussieu } \\
\text { (CFDT, CGT, FEN) }\end{array}$ \\
\hline 1975 & $\begin{array}{l}\text { Emergence de la première crise de l'amiante en } \\
\text { Organisation par le Collectif de Jussieu d'une rencontre médiatisée a }\end{array}$ \\
\hline 1976 & $\begin{array}{l}\text { Elargissement de la mobilisation. Mobilisation médiatique, dramatisation et diffusion des } \\
\text { enjeux de l'amiante. L'amiante n'est plus uniquement un problème industriel. Publication du } \\
\text { «Livre Blanc de l'amiante » par la Chambre syndicale de l'amiante et le Syndicat de } \\
\text { l'amiante-ciment, deux syndicats professionnels très puissants. }\end{array}$ \\
\hline 1977 & Publication par le Collectif de Jussieu de "Dan \\
\hline $\begin{array}{l}1977- \\
1978\end{array}$ & $\begin{array}{l}\text { Premier train de mesures : Mesure princip } \\
\text { bâtiments }+ \text { mesures de protections des prof } \\
\text { d'amiante autorisée, consigne de sécurité por }\end{array}$ \\
\hline \multicolumn{2}{|r|}{ Fin de la première crise de l'amiante en France } \\
\hline 1982 & $\begin{array}{l}\text { ymposium mondial sur l'amiante à Montréal organisé par les syndicats profe } \\
\text { miante }\end{array}$ \\
\hline 1982 & Création du CPA (Comité Perı \\
\hline \multirow[t]{2}{*}{$\begin{array}{c}1987- \\
1988\end{array}$} & $\begin{array}{l}\text { Traduction des directives européennes : Abaiss } \\
1988 \text { : Interdiction de l'amiante (excepté le chry }\end{array}$ \\
\hline & Emergence \\
\hline 1994 & $\begin{array}{l}\text { Plainte des veuves de professeurs de Gérardmer, Nouvel intérêt médiatique (publication de } 2 \\
\text { enquêtes journalistiques) Conférence à Paris VII organisée par le Comité anti-amiante de } \\
\text { Jussieu ; Présentation des travaux alarmistes de l'épidémiologiste britannique Julian Peto }\end{array}$ \\
\hline 1995 & Fin du CPA (septembre) \\
\hline \multirow[t]{2}{*}{1996} & $\begin{array}{l}\text { Création de l'ANDEVA (Association Nationale des Victimes de l'Amiante); Dépôt de } \\
\text { plaintes contre X par des victimes membres de l'ANDEVA, procédure pénale. Mise en place } \\
\text { d'une véritable stratégie juridique. Mobilisation médiatique et sociale : le cas de l'amiante } \\
\text { devient un scandale de santé publique. }\end{array}$ \\
\hline & $\begin{array}{l}\text { Nouvelles mesures législatives (vers l'interdiction): renforcement des mesures prises en } \\
\text { 1977-1978 (VLE, diagnostic amiante, protection des salariés). }\end{array}$ \\
\hline 1997 & \\
\hline
\end{tabular}

Figure 3 : Chronologie des principaux évènements de l'histoire de l'amiante en France

\section{Le Comité Permanent Amiante et l'Usage Contrôlé de l'Amiante en France}

\footnotetext{
${ }^{9}$ Un simple communiqué annonce que les membres du CPA ont décidé de ne plus y siéger et que le comité n'existe plus.

${ }^{10}$ La France est le 8ème pays européen à interdire totalement l'amiante. L'interdiction de l'amiante se fait comme dans tous les autres pays avec un calendrier de mise en œuvre et des dérogations.
} 


\section{La mise en place du CPA : entre doute scientifique et enjeux économiques}

Le mouvement précédent a été mené par des scientifiques qui avaient le savoir nécessaire pour créer une mobilisation autour de l'amiante. En effet, le collectif de Jussieu peut être perçu comme le pivot de la mobilisation de la fin des années 1970. Cette mobilisation disparait totalement : le collectif de Jussieu est dissout, la mission de porter l'amiante sur l'agenda politique est atteinte et les engagements politiques reposent sur un traitement rapide du dossier et la nécessité de mesures de protection ; l'entreprise Amisol est fermée et des plans de retour à l'emploi et de réinsertionsont prévus pour les anciens ouvriers; les associations attendent beaucoup des décisions politiques qui doivent protéger les citoyens, consommateurs et salariés. Ainsi, le premier train de mesures des années 1970 devait être en fait un premier pas vers l'interdiction de l'amiante en France. Cependant, les mesures prises à la fin des années 70 sont toutes relatives ${ }^{11}$ et n'évolueront plus pendant près de 10 ans suite à la mise en place du CPA et de l'usage contrôlé de l'amiante. Avant 1970, l'amiante était uniquement traité comme un risque professionnel au même titre que d'autres fibres. La mobilisation des années 70 a transformé cette vision. En effet, l'amiante n'est pas une fibre comme les autres. Elle est beaucoup plus nocive (les premières pathologies apparaissent). Elle est utilisée de manière intensive et peut donc toucher une population autre qu'ouvrière.Même si la dangerosité de l'amiante est avérée (reconnaissance comme produit cancérogène, pathologies développées, et même reconnaissance depuis 1945 au tableau des maladies professionnelles), le doute persiste sur les seuils d'exposition dangereux et les types d'amiante les plus nocifs ${ }^{12}$. La mobilisation n'a pas permis de répondre aux doutes qu'elle a soulevés. Ce doute permettra l'émergence d'une politique de gestion des risques dans un contexte

\footnotetext{
${ }^{11}$ Contrairement aux autres pays européens, il n'y a pas par exemple d'annonce réelle d'interdiction de l'amiante etle problème du désamiantage n'est pas du tout traité.

${ }^{12}$ Le débat sur la dangerosité des différents types d'amiante a été le sujet de nombreuses recherches dans les années 70. La crocidolite est reconnue pour être plus dangereuse et a été interdite en France en 1994. Le chrysotile est le type d'amiante le plus utilisé et celui qui est interdit en dernier.
} 
économique difficile. L'amiante est une matière première extrêmement efficace et peu coûteuse. C'est donc un facteur de compétitivité indéniable pour des industries comme l'automobile, la mécanique ou la construction. Secteurs déjà en difficulté et vivier d'emplois dans des régions françaises déjà marquées par la crise. L'usage d'un substitut apparaît à l'époque comme extrêmement coûteux et moins efficace. Face à l'absence de substitut évident, la question est alors de comprendre comment protéger la population des risques éventuels d'une exposition à l'amiante sans pour autant mettre en difficulté l'économie française. En 1982, quelques années donc après la fin de la mobilisation, le Comité Permanent Amiante est créé sous l'impulsion de l'Association Française de l'Amiante et de l'INRS (Institut National de Recherche et de Sécurité) avec pour objectif de comprendre et maîtriser l'amiante et surtout de protéger les personnes en limitant les risques d'exposition. Créé sous l'égide du Ministère des affaires sociales, le Comité Permanent Amiante est financé par les industriels de l'amiante (de manière indirecte et peu connue) et sa gestion est déléguée au cabinet de conseil «Communications économiques et sociale »(CES). Cette société de communication était alors dirigée par Marcel Valtat, aujourd'hui décédé. Le principe fondateur de cette organisation est de rassembler autour de la même table des représentants des industriels de l'amiante, des représentants des principaux syndicats, des spécialistes de la médecine du travail et des épidémiologistes.Le CPA est présenté comme une structure originale de concertation. Lors du symposium organisé à Montréal en 1982, Marcel Valtat présente cette innovation organisationnelle: " Le schéma traditionnel et rassurant selon lequel, dans un problème de ce type, il reviendrait aux scientifiques de dire le vrai, aux

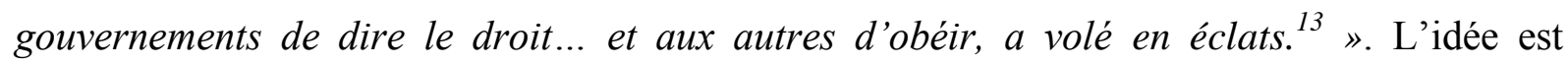
clairement de réunir autour de la table les différents acteurs de la précédente crise pour trouver «dans la transparence totale et la confiance retrouvée entre les hommes, des

\footnotetext{
${ }^{13}$ Amiante : où en est-on en France ? Journée d'étude du 9 novembre 1982 Compte rendu intégral des communications et des débats. Séance du matin M. VALTAT : le symposium mondial de Montréal
} 
solutions acceptables pour l'ensemble de la communauté. ${ }^{14} \gg$ Sont donc présents dans cet espace de discussion des représentants de divers horizons. Le tableau présenté ci-dessous (Figure 4) propose une synthèse des acteurs présents et leur motivation à participer au CPA.

\footnotetext{
${ }^{14}$ L'usage contrôlé de l'amiante. Utopie ou Réalité ? L'usage contrôlé de l'amiante, page 6, Publication du CPA
} par l'INRS, $3^{\text {ème }}$ édition, Mise à jour au $1^{\text {er }}$ janvier 1994 


\begin{tabular}{|c|c|c|}
\hline Catégories & Présentation & Objectifs affichés \\
\hline $\begin{array}{l}\text { Administrations } \\
\text { centrales de la Santé, } \\
\text { du Travail, de } \\
\text { l'Environnement et } \\
\text { de l'Industrie }\end{array}$ & $\begin{array}{l}\text { Des représentants des différents ministères concernés } \\
\text { par la question de l'amiante. (Ministères responsables } \\
\text { des questions de travail, de santé, d'écologie et de } \\
\text { logement) }\end{array}$ & $\begin{array}{l}\text { Le CPA est créé sous l'égide du Ministère de la santé en 1982. Avoir une } \\
\text { structure qui contrôle et maîtrise un dossier complexe. } \\
\text { Arbitrage entre doute scientifique et enjeux économiques. } \\
\text { Donner des garanties au niveau européen et international: forte crédibilité } \\
\text { et légitimité de cette structure multipartite. } \\
\text { Organiser la prise en charge du dossier. } \\
\text { Répondre aux attentes des différents acteurs économiques et sociaux. }\end{array}$ \\
\hline Organismes publics & $\begin{array}{l}\text { Des représentants de l'INRS, de différentes CRAM } \\
\text { (Caisse Régionale d'Assurance Maladie) et de } \\
\text { l'INSERM }\end{array}$ & $\begin{array}{l}\text { Rassembler les différents acteurs de différentes branches (santé au travail } \\
\text { et santé publique en particulier) } \\
\text { Obtenir des informations ; prévoir les différents risques d'exposition } \\
\text { Neutralité affichée du CPA } \\
\text { Caution scientifique }\end{array}$ \\
\hline $\begin{array}{l}\text { Industriels } \\
\text { (producteurs, } \\
\text { transformateurs et } \\
\text { utilisateurs) }\end{array}$ & $\begin{array}{l}\text { Des représentants des industries transformatrices de } \\
\text { l'amiante à travers l'Association Française de } \\
\text { l'Amiante (anciennement Chambre Syndicale de } \\
\text { l'Amiante et Syndicat de l'Amiante-Ciment) : deux } \\
\text { grands groupes (Eternit et Saint-Gobain) et leurs } \\
\text { filiales } \\
\text { Des représentants des entreprises utilisatrices de } \\
\text { l'amiante (automobile, BTP, construction navale en } \\
\text { particulier) }\end{array}$ & $\begin{array}{l}\text { Participer aux échanges et être informés des avancées et recherche en } \\
\text { cours } \\
\text { Défense des intérêts économiques }\end{array}$ \\
\hline $\begin{array}{l}\text { Confédérations } \\
\text { syndicales }\end{array}$ & $\begin{array}{l}\text { Les principaux syndicats sont représentés au sein du } \\
\text { CPA. (CFDT, CGT, CFE-CGC, CFDT) } \\
\text { (sortie du syndicat FO en } 1986 \text { suite à un différend } \\
\text { relatif au financement par l'INSERM d'une } \\
\text { conférence organisée par les industriels de l'amiante à } \\
\text { Montréal). }\end{array}$ & $\begin{array}{l}\text { Participer aux débats pour la défense des ouvriers } \\
\text { Sauvegarde de l'emploi : la France traverse une crise économique, il faut } \\
\text { préserver l'emploi industriel en France ; sans amiante, pas d'industrie } \\
\text { compétitive. Ce risque touche les entreprises transformatrices de l'amiante } \\
\text { (Eternit, Saint-Gobain et leurs filiales) mais aussi toutes les industries } \\
\text { utilisant de l'amiante (industrie automobilie, BTP, construction navale en } \\
\text { particulier). }\end{array}$ \\
\hline $\begin{array}{l}\text { Experts scientifiques } \\
\text { et médicaux }\end{array}$ & $\begin{array}{l}\text { Spécialistes en épidémiologie et médecine du travail } \\
\text { Des membres du mouvement précédent (en particulier } \\
\text { le Professeur Bignon) }\end{array}$ & $\begin{array}{l}\text { Participer à la réflexion scientifique. Rôle d'experts appelés par les } \\
\text { pouvoirs publics. Objectif : limiter les risques et protéger les personnes } \\
\text { exposées. Bénéficier de financements de recherche. }\end{array}$ \\
\hline $\begin{array}{l}\text { Représentant de } \\
\text { consommateurs }\end{array}$ & Institut National de la Consommation & Protéger et informer les consommateurs \\
\hline
\end{tabular}

Figure 4 Acteurs présents au CPA et objectifs affichés de cette participation 
Cette collaboration n'aurait sûrement pas été la même si le postulat de départ du CPA avait été différent. Chaque acteur présent devait alors répondre à la question : comment utiliser l'amiante sans mettre les personnes en danger. Dès lors, les médecins ont pu adopter un rôle d'experts-conseils pour faire des préconisations et donner des guides de conduite aux industriels, les syndicats quant à eux protégeaient l'emploi $^{15}$ de régions dévastées ${ }^{16}$. Le consensus établit sera tout de même fragilisé par la sortie du syndicat FO du Comité suite à un différend relatif au financement par l'INSERM d'une conférence organisée par les industriels de l'amiante à Montréal. Le syndicat FO décidera de sortir du CPA. Le Professeur Pézerat et d'autres représentants du premier collectif de Jussieu refuseront quant à eux la collaboration proposée et ne siègeront jamais au CPA. Absents de la structure de décision, et dépourvus de soutiens, ils seront marginalisés et donc absents des débats. ${ }^{17}$ Le choix organisationnel (présence d'acteurs légitimes et indépendants) et ses objectifs (double objectif économique et social) confèrent au CPA une légitimité qui empêche la contestation. Le système CPA est par ailleurs largement verrouillé : les journalistes qui s’y sont intéressés dans les années 80 se sont heurtés à une communication contrôlée et une organisation au final assez opaque ${ }^{18}$.

\section{La promotion de l'usage contrôlé de l'amiante}

L'usage contrôlé de l'amiante est tout naturellement devenu la nouvelle ligne de conduite sur le dossier de l'amiante. L'usage contrôlé de l'amiante est alors devenu la vitrine de la bonne gestion de l'amiante et a été promu par les différents membres au niveau international (ainsi, les Professeurs Bignon et Brochard ont été dans de nombreux pays pour faire des conférences sur le thème : Brésil, Canada, Tunisie entre autres pays).L'usage contrôlé de l'amiante part

\footnotetext{
${ }^{15}$ La question des conditions de travail était alors assez secondaire dans les luttes menées par les syndicats. L'emploi était l'enjeu essentiel et les risques professionnels étaient alors beaucoup plus acceptés.

${ }^{16}$ Les entreprises transformatrices d'amiante étaient très implantées dans le Nord de la France et dans l'Est. L'histoire d'Eternit est à ce propos extrêmement révélateur (voir les travaux de O. Hardy-Hémery).

${ }^{17}$ Les « années CPA » sont marquées par l'absence d'actions anti-CPA. Cela s'explique notamment par un manque d'accès à des données et aussi l'absence de " preuves ", les pathologies ne s'étant pas encore toutes révélées.

${ }^{18}$ A titre d'exemple, son financement par les industriels de l'amiante était largement méconnu, seule prévalait la caution des pouvoirs publics.
} 
d'un constat relativement simple : l'homme est entouré de matières naturelles dangereuses qu'il a appris à contrôler. L'amiante appartient à cette catégorie de «matériaux dangereux mais néanmoins utiles ${ }^{19}$. Il faut pour «l'avantage collectif» apprendre à se protéger et réduire les effets néfastes par des politiques de protection et des évaluations régulières de ces mesures. La possibilité d'avoir recours à des matériaux de substitution n'est pas écartée mais pour cela il doit répondre à plusieurs critères : il doit être efficace, moins risqué et... «économiquement acceptable $»^{20}$ Ce point sera sujet à controverse après l'interdiction de l'amiante et ouvre la voie à une analyse plus critique du CPA.

\section{Les limites du CPA}

En redéfinissant la pratique, le CPA a joué davantage qu'un rôle de structure de collaboration et d'échange. En effet, en instaurant l'usage contrôlé de l'amiante comme un nouveau dogme, le CPA a participé très fortement au maintien de l'usage de l'amiante. Finalement, il n'y a pas eu de changements radicaux dans la pratique et les rapports et enquêtes menés a posteriori ont montré que les préconisations en termes de sécurité et de protection ne s'étaient pas toujours traduites en pratiques $^{21}$. La première limite du CPA semble être là : comment s'est concrètement traduit l'Usage Contrôlé de l'Amiante ? La dédramatisation de l'amiante a conduit aussi au fait que les ouvriers exposés ne se sentent pas concernés par les risques liés à l'amiante. Il y a eu un décalage entre le discours et les bonnes pratiques conseillées et leur mise en œuvre. Le rôle de la médecine et de l'inspection du travail a ici joué un rôle central ${ }^{22}$. Seconde limite, comme souligné précédemment, il y a des absents dans le CPA. Premiers absents, le Professeur Pézerat et tous les autres acteurs de la précédente mobilisation qui n'ont

\footnotetext{
${ }^{19}$ L'usage contrôlé de l'amiante. Utopie ou Réalité ? L'usage contrôlé de l'amiante, page 11, Publication du CPA par l'INRS, $3^{\text {ème }}$ édition, Mise à jour au $1^{\text {er }}$ janvier 1994

${ }^{20}$ Ce point est celui qui reste encore le plus contestable, car il n'existe encore aujourd'hui aucun substitut qui puisse à répondre à ces trois critères simultanément. La notion d'acceptable est largement subjective.

${ }^{21}$ Des entretiens avec d'anciens ouvriers de Renault ont confirmé le manque de connaissance des risques liés à l'amiante. Fin des années, des archives montrent des salariés de l'entreprise en train de vider un entrepôt d'amiante à mains nues, sans protection.

${ }^{22}$ La question de la sécurité au travail dépasse largement la question de l'amiante et du CPA.
} 
pas adhéré au compromis et restaient persuadés qu'il ne fallait pas collaborer avec les industriels pour traiter d'un sujet de santé publique. Ces acteurs n'ont alors pas eu accès aux financements pour leurs travaux et leurs publications ne bénéficiaient pas d'une légitimité suffisante pour discuter les travaux publiés par les Professeurs, membres du CPA. Le CPA a publié de nombreux avis négatifs relatifs aux recherches françaises et internationales. Interlocuteur privilégié des pouvoirs publics, ils ont ainsi participé au maintien de la pratique. Autres absents, les «industriels hors amiante». Ce point est central dans la nouvelle définition de l'usage contrôlé de l'amiante. Nous avons souligné précédemment les restrictions posées à la recherche de substitut. Ces industries ne siégeant pas au CPA pouvaient difficilement proposer leur solution. Difficile pour les médecins et autres représentants syndicaux de mettre en perspective les arguments des industriels de l'amiante ${ }^{23}$. Enfin, difficile de dissocier le CPA de la conférence internationale organisée par les industriels de l'amiante en 1971 à Londres pour préparer les difficultés à venir. Les membres de la Chambre Syndicale de l'Amiante et du Syndicat de l'Amiante-Ciment ont assisté à cette conférence et semble avoir appliqué à la lettre les préconisations.

\section{Extrait du commentaire final de la conférence de Londres (Novembre 1971)}

- « Je ne peux donc que vous inviter très sérieusement à vous préparer dès maintenant à faire face à une plus grande intervention des autorités publiques et à des attaques de plus en plus violentes ॥ ;

- « je vous invite tous à préparer votre défense dès maintenant. [...] avez-vous un comité d'action disposant des fonds nécessaires, mais aussi d'une expertise technique et médicale ? [...] êtes-vous en contact avec des consultants en relations publiques capables de vous donner de bons conseils?";

- « la maxime « ne réveillez pas le chat endormi » est tout à fait appropriée lorsque les choses vont lentement et que l'intérêt du public et de la presse reste faible. Mais les chats endormis peuvent se réveiller brutalement, faire entendre leur voix et montrer leurs griffes. [...] Vous devez vous préparer à l'avance ".

Figure 5 : Extrait du commentaire final de la conférence de Londres (Novembre 1971)

\footnotetext{
${ }^{23}$ Lors d'un entretien, le Professeur Brochard, membre du CPA a souligné à plusieurs reprises qu'il n'était pas ingénieur et qu'en participant au CPA il ne faisait que répondre à la question qu'on lui posait : comment protéger les ouvriers des risques liés à l'amiante?
} 
Les syndicats professionnels de l'amiante ont participé à l'élaboration des lois de 1977 comme le préconise le rapport. En finançant le CPA, ils ont créé un lieu d'expertise technique et médicale. Le cabinet CES est spécialisé en communication et relation presse et le CPA a totalement contrôlé les débats sur la période 1982-1992, dénigrant les résultats d'études menées à l'international. Le poids politique du CPA est indéniable.La structure opaque qui entourait le CPA et la ressemblance avec les conclusions de cette conférence participent à la mise en perspective des objectifs affichés par les industriels quant à la participation à cette organisation.Le cas de l'amiante est complexe à traiter tant au niveau scientifique que technique. Pour preuve, les réglementations adoptées prévoient des délais d'adaptation et de transition sur plusieurs années. Encore aujourd'hui les débats subsistent sur le bien-fondé d'une interdiction totale ${ }^{24}$. Le CPA a été construit dans un contexte particulier: crise économique et incertitude scientifique. Dès lors l'arbitrage entre risques de santé publique et enjeux économiques a été particulièrement complexe. Il était particulièrement difficile d'évaluer les risques associés à l'amiante en sachant que les pathologies n'étaient pas encore toutes déclarées. Dans le cadre du CPA, les enjeux économiques ont largement prédominé puisqu'ils ont défini les contours de l'usage de l'amiante. Les acteurs économiques ont bénéficié de la légitimité conférée par le CPA. Indépendamment, les industriels auraient sûrement eu moins d'influence dans les prises de décision liées à l'amiante en France.

\section{DISCUSSION}

Notre travail offre un nouveau regard sur la stratégie de maintien institutionnel issue du travail défensif engagé par les acteurs bénéficiant de la pratique. La mise en place d'une organisation frontière peut être dans un contexte particulier une stratégie de défense qui permet de sortir d'une phase de contestation. En effet, la mise en place d'une organisation

\footnotetext{
${ }^{24}$ Aujourd'hui encore le cas du désamiantage fait débat : vaut-il mieux désamianter à coût élevé au risque d'exposer riverains et désamianteurs soumis à la poussière produite, ou serait-il suffisant de calfeutrer l'amiante à moindre coût et mettre en place un système de vigilance efficient ?
} 
frontière a permis aux acteurs de proposer une nouvelle pratique qui permet de fédérer des acteurs aux intérêts pourtant divergents. Maguire, Hardy et Lawrence (2004) ont dans leur travail sur la lutte contre le sida montré comment les activistes avaient participé à la création d'une nouvelle communauté associant régulateurs, firmes pharmaceutiques et soignants afin d'influencer les décisions politiques. On retrouve ici la même dynamique avec la mise en place d'une organisation frontière. La mise en place du CPA en tant qu'organisation frontière a donné suffisamment de légitimité à l'institution pour imposer une nouvelle logique institutionnelle (Friedland et Alford, 1991). L'usage contrôlé de l'amiante a permis de revoir la pratique institutionnalisée et de redéfinir ce qu'il faut faire ou ne pas faire dans le champ. Comme le soulignent Zietsma etLawrence (2010), quand les frontières du champ et la pratique sont contestées, les acteurs doivent innover en construisant de nouvelles frontières qui les protègent à nouveau de la sanction institutionnelle. A ce titre, la mise en œuvre d'une organisation frontière peut être perçue comme une innovation institutionnelle. En mettant en place une telle structure, les industriels de l'amiante ont utilisé leur capacité d'agence pour maintenir la pratique. Il y a donc bien eu un travail sur les frontières (boundarywork)puis une redéfinition de la pratique (practice work). Plus précisément, la redéfinition des frontières ont permis de marginaliser les acteurs contestataires ou de les intégrer et donc de limiter leur capacité de contestation en réduisant leur capacité d'agence. Ne pouvant plus contester la pratique ou les frontières, leur capacité d'agence est limitée à la reproduction des éléments de l'institution avec une capacité d'action plus proche de l'action routinière que de la capacité à questionner les fondements de la pratique.Le maintien institutionnel a souvent été perçu comme une conséquence d'un mécanisme d'isomorphisme et d'auto-reproduction des institutions. Les chercheurs se sont ainsi largement concentrés sur les régularités et le conformisme social (Zucker, 1988). Le cas de l'amiante et du CPA participe à illustrer une autre perspective du maintien institutionnel : la reproduction des règles et des croyances est le 
fruit d'un engagement des acteurs dans un travail institutionnel de maintien (Blanc et Huault, 2010 ; Lawrence et Suddaby, 2006). La mise en œuvre d'une organisation frontière participe à ce travail de maintien institutionnel. Issue d'un effort de collaboration, le CPA a permis le maintien de l'usage de l'amiante. L'originalité de la structure lui a conféré une légitimité dans un contexte particulier. L'intelligence des industriels, à l'origine de cette organisation, est d'avoir réussi à proposer une nouvelle définition de la pratique qui réponde à ces pressions extérieures, tout en conservant les éléments principaux de la pratique institutionnalisée. Durand, Rao, Monin (2007) ont ainsi mis en évidence l'impact des changements de code sur l'évaluation par un tiers dans la haute cuisine française et l'opposition entre classique et nouvelle cuisine française. Ils s'appuient ainsi sur les concepts de «code-preserving changes » (c'est-à-dire des changements dans les codes qui ne modifient pas l'ordre social existant) et «code-violating changes » (qu'ils définissent comme les changements qui rompent avec l'ordre social précédent). Comme le soulignent les auteurs, l'évaluation de ces changements est corrélée à une mesure relative du changement proposé. En l'occurrence, le cas proposé ici ne repose pas sur une évaluation externe par un tiers mais davantage sur la question de l'adhésion à la pratique au sein de l'organisation frontière qui assure sa légitimité. Les acteurs concèdent ainsi une reconnaissance du risque associé à la pratique (les industriels), sans pour autant remettre en cause le fondement de l'utilité économique et social de la pratique (les médecins et représentants syndicaux).

A travers l'étude du CPA, nous avons également pu répondre à l'appel à comprendre les moments de collaboration O’Mahony et Bechky (2008, p455) «It isimperativethatwedevelop a framework for understanding not only how contestation ismobilized but how itistransformed ». L'organisation frontière permet d'être une réponse à une situation de crise et de contestation. La sortie de crise était amorcée par la mise sur l'agenda politique de la question de l'amiante. Le CPA met en exergue la capacité des acteurs, que l'on pourrait 
qualifier d' « unexpected allies »,à collaborer dans un but commun (Giugni, 1998 ; Earl, 2004). Cette capacité à collaborer dépend des intérêts convergents déterminés lors de la constitution de la structure. L'étude du CPA met en évidence l'évolution des équilibres. En l'occurrence ici les industriels de l'amiante en sortent largement bénéficiaires : l'usage de l'amiante a été maintenu et la structure du CPA a allégé le poids de leur responsabilité individuelle dans le drame de l'amiante. O’Mahony et Bechky (2008) soulignent assez peu le rôle de la composition de l'organisation frontière et des pressions institutionnelles sur la définition des intérêts convergents et divergents. L'organisation frontière crée des frontières particulièrement étanches qui biaisent la définition de l'objectif partagé. Ainsi, l'absence de représentants de victimes ou de représentants d'autres secteurs de l'industrie substituts ou complémentaires a limité les perspectives d'agence en ne proposant pas de modèles alternatifs et a donc participé au maintien institutionnel. C'est sûrement dans une perspective plus critique l'aspect négatif de la mise en place d'une telle organisation frontière dans un contexte de doutes scientifiques et de tensions économiques. Dans ce contexte, l'organisation frontière est alors un moyen pour les entreprises de se prémunir des incursions d'éventuels compétiteurs en protégeant leur corps de métier (Bechky, 2003). Ici, les industriels ont par exemple concéder l'abandon du flocage mais conserver tous les autres usages tout en excluant l'accès à toute autre entreprise. L'organisation frontière s'inscrit alors tout à fait dans un travail de maintien institutionnel sur les frontières institutionnelles (boundarywork).

\section{Perspectives et Voies de recherche}

L'étude du CPA comme organisation frontière s'inscrit dans la trajectoire plus large de la désinstitutionalisation de l'amiante en France. L'existence de cette organisation est le fruit de la réussite du travail défensif mis en place par les industriels de l'amiante dans les années 80. Dans leur travail sur le DDT, Maguire et Hardy (2009) ont ainsi mis en évidence les stratégies discursives mises en œuvre par les acteurs suite à la publication du pamphlet 
«SilentSpring »qui a dénoncé les risques pour l'environnement et la santé de l'usage de ce pesticide. Le cas du DDT soulignait le rôle d'un choc externe comme moteur de désinstitutionalisation. Dernière étape du cycle de vie des institutions, la désinstitutionalisation, c'est-à-dire le processus par lequel une pratique prise pour acquise est totalement délégitimée (Oliver, 1992), est pourtant encore mal connue. La dissolution du CPA suite à la ré-émergence d'un mouvement socialdans les années 90 marque le début à proprement parler de la désinstitutionalisation. La stratégie mise en œuvre par les acteurs marginalisés va alors s'appuyer sur une stratégie juridique originale et la dénonciation d'un scandale de santé publique. Ce travail de destruction de la pratique pourrait faire l'objet d'une étude approfondie. Plus particulièrement, la question de la rupture du consensus reste encore à explorer. Le cas de l'amiante permet de mettre en perspective deux périodes de contestations portées par un mouvement. L'une ayant conduit à une période de maintien institutionnel et l'autre à une période de destruction. La redéfinition des frontières du champ par la mise en place d'une organisation frontière a permis aux acteurs de maintenir la pratique mais le travail engagé pour maintenir une pratique peut alors être insuffisant quand les conditions sociales extérieures au champ évoluent et que les acteurs externes s'engagent dans un travail de destruction de la pratique (Zietsma et Lawrence, 2009).

L'usage contrôlé de l'amiante a été le résultat du consensus en France. Forte de cette nouvelle doctrine, la France a exporté ce modèle dans de nombreux pays comme le Canada ou le Brésil. Sans surprise, ces pays sont producteurs d'amiante (et exportateurs vers de nombreux pays en voie de développement). Il serait alors intéressant de confronter le cas de la France à un niveau international. L'étude des institutions au niveau international offrirait une perspective intéressante de poursuite de cette étude. La dimension culturelle jouerait alors un rôle central dans la transposition de la pratique. L'internationalisation porte tout autant sur l'usage contrôlé de l'amiante que sur le mouvement social. On assiste en effet à une 
internationalisation du mouvement anti-amiante («Ban Asbestos» est également issu du mouvement créé par le Collectif de Jussieu et est actuellement extrêmement actif sur la scène internationale) et à un renforcement de la position des industriels dans nombre de ces pays.

\section{BIBLIOGRAPHIE}

Arndt M., Bigelow B., "Professionalizing and masculinizing a female occupation: The reconceptualization of hospital administration in the early 1900s", Administrative Science Quartely, vol.50, 2005, p. 233-261

Barley S.R., Tolbert P.S., "Institutionalization and Structuration: Studying the Links Between Action and Institution”, Organization Studies, vol. 18, N 1, 1997, p. 93-117.

Barnes B., "Practice as collective action", in Schatzi T.R., Knorr Cetina K., Von Savigny E., eds, The Practice Turn in Contemporary Theory, London: Routledge, 2001, p. 17-28.

Bechky B.A., "Object lessons: Workplace artifacts as representations of occupational jurisdiction”, American Journal of Sociology, vol. 109, 2003, p. 720-752.

Blanc A., Huault I., "Reproduction de l'ordre institutionnel face à l'incertitude", Revue française de gestion, vol. 36, $\mathrm{n}^{\circ} 203$, avril 2010, p. 85-99.

Dériot G., Godefroy J. P., Le drame de l'amiante en France : comprendre, mieux réparer, en tirer des leçons pour l'avenir, Les Rapports du Sénat, n³ 37, 2005-2006.

Durand R., Rao H., Monin P., "Code and conduct in French cuisine: impact of code changes on external evaluations”, Strategic Management Journal, vol. 28, 2007, p. 455-472.

Fligstein N., "Social Skill and the Theory of Fields", Sociological Theory, vol. 19, № 2 , 2001, p. 105-125.

Friedland R., Alford R.R., "Bringing Society Back in: Symbol, Practices, and Institutional Contradictions." W. W. Powell, P.J. DiMaggio, eds. The new institutionalism in organizational analysis, University of Chicago Press, Chicago, 1991, p. 232-263.

Giugni M. G.,"Was it worth the effort? The outcomes and consequences of social movements", Annual Review of Sociology, vol. 98, 1998, p.371-393.

Guston D.H., "Stabilizing the boundary between US politics and science: The role of the office of Technology Transfer as a boundary organization", Social Studies of Science, vol. 29, 2001, p. 87-111.

Jackall R., Moral Mazes: The World of Corporate Managers, Oxford University Press, New York, 1988.

Jepperson R.L., "Institutions, Institutional Effects, and Institutionalism”. W.W. Powell, P.J. DiMaggio, éds. The New Institutionalism in Organizational Analysis, University of Chicago Press, Chicago, 1991, p. 143-163.

Lawrence T.B., Hardy C., "Building bridges for refugees: toward a typology of bridging organizations", Journal of Applied Behavioral Science, vol. 35, 1999, p. 48-70. 
Lawrence T.B., Suddaby R., "Institutions and Institutional Work", S. Clegg, éd.The Sage handbook of organization studies, Sage Publications, London, Tousand Oaks, Calif, 2006, p. 215-254

Lawrence T.B, Suddaby R., Leca B., Institutional Work: Actors and Agency in Institutional Studies of Organizations, Cambridge University Press, 2009, p 1-27.

Maguire S., Hardy C., "Discourse and deinstitutionalization: the decline of ddt",Academy of Management Journal,vol. 52, ํㅜ 1, February 2009, p. 148-178.

Maguire S., Hardy C., Lawrence T.B., "Institutional entrepreneurship in emerging fields: HIV/AIDS treatment advocacy in Canada", Academy of Management Journal, vol.47, 2004, p. 657-679.

Malye F., Amiante, le dossier de l'air contaminé, Le Pré aux Clercs, Paris, 1996.

Oliver C., «The Antecedents of Deinstitutionalization », Organization Studies, vol. 13, 1992, p. 563-588.

O’Mahony S., Bechky B. A., "Boundary Organizations:Enabling Collaboration among Unexpected Allies", Administrative Science Quartely, 53, 2008, p 422-459.

Scott W.R., Institutions and organizations, Sage Publications, Thousand Oaks, Calif., 2001.

Snow D. A., Soule S. A., Kriesi H., "Mapping the terrain" In D. A. Snow, S. A. Soule, and H. Kriesi (eds.), The Blackwell Companion to Social Movements, Malden, MA: WileyBlackwell, 2004, p. 3-16.

Whittington R., "Completing the practice turn in strategy research", Organization Studies, vol. 27, 2006, p. 613-634.

Yin R.K., Case study research: Design \& Methods, Sage Publications, Thousand Oaks, Calif., 2003.

Zietsma C., Lawrence T.B., "Institutional Work in the Transformation of an Organizational Field: The Interplay of Boundary Work and Practice Work", Administrative Science Quartely, vol. 55, 2010, p. 189-221.

Zucker L.G., "Institutional Theories of Organization”, Annual Review of Sociology, vol. 13, $\mathrm{N}^{\circ} 1,1987$, p. 443-464.

Zucker L.G., "Where do institutional patterns come from ?Organizations as actors in social systems", Institutional patterns and organizations, Zucker L.G., éd, MA, Ballinger, Cambridge, 1988, p. 23-52. 